\title{
A NOTE ON LIGHTLIKE HYPERSURFACES OF SEMI-RIEMANNIAN SPACE FORM
}

\author{
YANING WANG AND XIMIN LIU
}

\begin{abstract}
In this paper, we mainly study lightlike hypersurfaces of semi-Riemannian space form. Our main result is a classification theorem of screen conformal lightlike hypersurfaces. Also, we obtain some geometric properties of lightlike hypersurfaces with a conformal Killing distribution.
\end{abstract}

\section{INTRODUCTION}

Since the intersection of tangent bundle and the normal bundle of a lightlike submanifold is not trivial, there are more difficulties in studying lightlike geometry than in the non-degenerate case. It is well known that lightlike geometry have been studied by K. L. Duggal and A. Bejancu $[2,3]$ and D. N. Kupeli [9] with different approaches. Recently, many geometers investigated lightlike hypersurfaces by using the fundamental knowledge introduced by Duggal-Bejancu with various geometric conditions and obtained many important results. For example, D. H. Jin [6] proved a classification theorem of lightlike hypersurface $\mathrm{M}$ with totally umbilical screen distribution of a semi-Riemannian space form; C. Atindogbe and K. L. Duggal [1] introduced screen conformal lightlike hypersurface and proved that its induced Ricci curvature tensor is symmetric. For more related results in this field, we refer the readers to the recent monographs by Duggal-Jin [4] and Duggal-Sahin [5], respectively.

In this paper, we mainly study a screen conformal lightlike hypersurface $M$ immersed in a semi-Riemannian space form and obtain a classification theorem of $M$. Also, we investigate lightlike hypersurface with a conformal

2010 Mathematics Subject Classification. 53C25, 53C50.

Key words and phrases. Lightlike hypersurface, semi-Riemannian space form, screen conformal, conformal Killing distribution.

The project is supported by Natural Science Foundation of Guangdong Province of China (Project No. S2011010000471) and NSFC (10931005). 
Killing radical distribution and transversal distribution respectively which were introduced by D. H. Jin and J. W. Lee [8].

\section{Preliminaries}

In this section, we follow [3] developed by Duggal-Bejancu for the notations and fundamental equations for lightlike hypersurface of semi-Riemannian manifolds.

A submanifold $(M, g)$ of dimension $m$ immersed in a semi-Riemannian manifold $(\bar{M}, \bar{g})$ of dimension $(m+n)$ is called a lightlike submanifold if the metric $g$ induced from ambient space is degenerate and the radical distribution $\operatorname{Rad}(T M)$ is of rank $r$, where $m \geq 2$ and $1 \leq r \leq n$. In particular, $(M, g)$ is called a lightlike hypersurface if $n=1$ and $r=1$. It is well known that the radical distribution is given by $\operatorname{Rad}(T M)=T M \cap T M^{\perp}$, where $T M^{\perp}$ is called the normal bundle of $M$ in $\bar{M}$. Thus there exists a nondegenerate complementary distribution $S(T M)$ of $\operatorname{Rad}(T M)$ in $T M$, which is called the screen distribution on $M$. Thus we have

$$
T M=\operatorname{Rad}(T M) \oplus_{\text {orth }} S(T M),
$$

where $\oplus_{\text {orth }}$ denotes the orthogonal direct sum. We denote such a lightlike hypersurface by $(M, g, S(T M))$.

Theorem 2.1. [3] Let $(M, g, S(T M))$ be a lightlike hypersurface of a semiRiemannian manifold $(\bar{M}, \bar{g})$, Then there exists a unique lightlike vector bundle $\operatorname{tr}(T M)$ of rank 1 over $M$, such that for any non-zero section $\xi$ of $T M^{\perp}$ on a coordinate neighborhood $\mathcal{U} \subset M$, there exists a unique section $N$ of $\operatorname{tr}(T M)$ on $\mathcal{U}$ satisfying:

$$
\bar{g}(N, \xi)=1, \quad \bar{g}(N, N)=\bar{g}(N, W)=0, \quad \forall W \in \Gamma\left(\left.S(T M)\right|_{\mathcal{U}}\right) .
$$

Hence for any screen distribution $S(T M)$ we have a unique bundle $\operatorname{tr}(T M)$ which is the complementary vector bundle to $T M$ in $\left.T \bar{M}\right|_{M}$ and satisfies $(2,2)$. Then we have the following decompositions:

$$
\left.T \bar{M}\right|_{M}=S(T M) \oplus_{\text {orth }}(\operatorname{Rad}(T M) \oplus \operatorname{tr}(T M))=T M \oplus \operatorname{tr}(T M) .
$$

We call $\operatorname{tr}(T M)$ and $N$ the transversal vector bundle and the null transversal vector field of $M$ with respect to $S(T M)$ respectively.

Let $P$ be the projection morphism of $T M$ on $S(T M)$ with respect to the decomposition (2.1) and $\bar{\nabla}$ be the Levi-Civita connection of $\bar{M}$. For any $X, Y \in \Gamma(T M), N \in \Gamma(\operatorname{tr}(T M))$ and $\xi \in \Gamma(\operatorname{Rad}(T M))$, the Gauss and Weingarten formulas of $M$ and $S(T M)$ are given by

$$
\begin{aligned}
& \bar{\nabla}_{X} Y=\nabla_{X} Y+B(X, Y) N, \\
& \bar{\nabla}_{X} N=-A_{N} X+\tau(X) N,
\end{aligned}
$$


and

$$
\begin{aligned}
\nabla_{X} P Y & =\nabla_{X}^{*} P Y+C(X, P Y) \xi, \\
\nabla_{X} \xi & =-A_{\xi}^{*} X-\tau(X) \xi,
\end{aligned}
$$

respectively, where $\nabla$ and $\nabla^{*}$ are the induced connections on $T M$ and $S(T M)$ respectively, $B$ and $C$ are called locally second fundamental forms of $M$ and $S(T M)$ respectively. $A_{N}$ and $A_{\xi}^{*}$ are linear operators on $T M$ and $S(T M)$ respectively and $\tau$ is a 1 -form on $T M$ defined by $\tau(X)=\bar{g}\left(\bar{\nabla}_{X} N, \xi\right)$. Since $\bar{\nabla}$ is a torsion-free and metric connection on $\bar{M}$, it is easy to see that $B$ is symmetric and independent of the choice of a screen distribution and satisfies

$$
B(X, \xi)=0, \quad \forall X \in \Gamma(T M) .
$$

Denote a local 1-form $\eta$ by $\eta(X)=g(X, N)$ for all $X \in \Gamma(T M)$, then the induced metric $g$ on $M$ satisfies

$$
\left(\nabla_{X} g\right)(Y, Z)=B(X, Y) \eta(Z)+B(X, Z) \eta(Y), \quad \forall X, Y, Z \in \Gamma(T M),
$$

which means that $\nabla$ is not a metric connection on $M$. But a simple computation implies that $\nabla^{*}$ is a metric connection on $S(T M)$. The above local second fundamental forms $B$ and $C$ of $M$ and $S(T M)$ are related to their shape operators by

$$
\begin{gathered}
B(X, Y)=g\left(A_{\xi}^{*} X, Y\right), \quad \bar{g}\left(A_{\xi}^{*} X, N\right)=0, \\
C(X, P Y)=g\left(A_{N} X, P Y\right), \quad \bar{g}\left(A_{N} X, N\right)=0,
\end{gathered}
$$

for any $X, Y \in \Gamma(T M)$ and $N \in \Gamma(\operatorname{tr}(T M))$.

From the above equations we see that $A_{\xi}^{*}$ and $A_{N}$ are $\Gamma(S(T M))$-valued shape operators related to $B$ and $C$ respectively, and $A_{\xi}^{*}$ is self-adjoint on $T M$ such that

$$
A_{\xi}^{*} \xi=0
$$

Donate by $\bar{R}, R$ and $R^{*}$ the curvature tensor of semi-Riemannian connection $\bar{\nabla}$ of $\bar{M}$, the induced connection $\nabla$ on $M$ and the induced connection $\nabla^{*}$ on $S(T M)$ respectively, we obtain the following Gauss-Codazzi equations for $M$ and $S(T M)$.

$$
\begin{gathered}
\bar{g}(\bar{R}(X, Y) Z, P W) \\
=g(R(X, Y) Z, P W)+B(X, Z) C(Y, P W)-B(Y, Z) C(X, P W) \\
\bar{g}(\bar{R}(X, Y) Z, \xi)=g(R(X, Y) Z, \xi) \\
=\left(\nabla_{X} B\right)(Y, Z)-\left(\nabla_{Y} B\right)(X, Z)+B(Y, Z) \tau(X)-B(X, Z) \tau(Y), \\
\bar{g}(\bar{R}(X, Y) Z, N)=g(R(X, Y) Z, N)
\end{gathered}
$$




$$
\begin{aligned}
& \bar{g}(\bar{R}(X, Y) P Z, N)=\left(\nabla_{X} C\right)(Y, P Z)-\left(\nabla_{Y} C\right)(X, P Z) \\
&+C(X, P Z) \tau(Y)-C(Y, P Z) \tau(X) \\
& \bar{g}(\bar{R}(X, Y) P Z, P W)=g\left(R^{*}(X, Y) P Z, P W\right) \\
&+B(Y, P W) C(X, P Z)-B(X, P W) C(Y, P Z)
\end{aligned}
$$

for all $X, Y, Z, W \in \Gamma(T M), \xi \in \Gamma(\operatorname{Rad}(T M))$ and $N \in \Gamma(\operatorname{tr}(T M))$.

\section{SCREen CONFORMAL LightLIKe hypersurfaces}

In this section, we consider a class of lightlike hypersurface which is called screen conformal lightlike hypersurface, defined as following.

Definition 3.1. [1] A lightlike hypersurface $(M, g, S(T M))$ of a semi-Riemannian manifold is called screen locally conformal if the shape operators $A_{N}$ and $A_{\xi}^{*}$ of $M$ and $S(T M)$ respectively, are related by

$$
A_{N} X=\varphi A_{\xi}^{*} X, \quad \forall X \in \Gamma(T M),
$$

where $\varphi$ is a non-vanishing smooth function on a neighborhood $\mathcal{U}$ in $M$. In particular, $M$ is said to be screen locally homothetic if $\varphi$ is non-zero constant.

In case $\mathcal{U}=M$ the screen conformality is global. In the sequel, by a screen conformal we shall mean screen locally conformal unless otherwise specified. Notice that by (2.10) and (2.11) we know that $M$ is said to be screen conformal if and only if

$$
C(X, P Y)=\varphi B(X, Y), \quad \forall X, Y \in \Gamma(T M) .
$$

Let $(M, g, S(T M))$ be a lightlike hypersurface of a semi-Riemannian manifold. Then, $S(T M)$ is said to be totally umbilical if on any coordinate neighborhood $\mathcal{U} \subset M$, there exists a smooth function $\gamma$ such that $A_{N}=\gamma P X$ for any $X \in \Gamma(T M)$, or equivalently,

$$
C(X, P Y)=\gamma g(X, Y), \quad \forall X, Y \in \Gamma(T M) .
$$

In case $\gamma=0$ on $\mathcal{U}, S(T M)$ is said to be totally geodesic.

Lemma 3.2. [6] Let $(M, g, S(T M))$ be an $(m+1)(m>2)$-dimensional lightlike hypersurface of a semi-Riemannian space form $(\bar{M}(c), \bar{g})$ such that $S(T M)$ is totally umbilical. Then $C=0$ or $B=0$. Moreover,

(1) $C=0$ and hence $S(T M)$ is totally geodesic and $c=0$, or

(2) $B=0$ and hence $M$ is totally geodesic immersed in $\bar{M}(c)$ and the induced connection $\nabla$ on $M$ is metric. 
Differentiating (3.2) gives

$$
\left(\nabla_{X} C\right)(Y, P Z)=X(\varphi) B(Y, P Z)+\varphi\left(\nabla_{X} B\right)(Y, P Z) .
$$

Substituting (2.14) and (2.16) into (3.3), we have

$$
\begin{aligned}
& \bar{g}(\bar{R}(X, Y) P Z, N)-C(X, P Z) \tau(Y)+C(Y, P Z) \tau(X) \\
= & X(\varphi) B(Y, P Z)-Y(\varphi) B(X, P Z)+\varphi \bar{g}(\bar{R}(X, Y) Z, \xi) \\
& -\varphi B(Y, Z) \tau(X)+\varphi B(X, Z) \tau(Y) .
\end{aligned}
$$

Notice that $\bar{M}$ is a semi-Riemannian space form, it follows from (2.10) and (3.2) and the above equation that

$$
\begin{aligned}
& B(X, Z)(Y(\varphi)-2 \varphi \tau(Y))-B(Y, Z)(X(\varphi)-2 \varphi \tau(X)) \\
= & c \eta(Y) g(X, Z)-c \eta(X) g(Y, Z), \quad \forall X, Y \in \Gamma(T M) .
\end{aligned}
$$

Replacing $Y$ by $\xi$ in the above equation and using (2.10), we have

$$
\beta B(X, Y)=c g(X, Y), \quad \forall X, Y \in \Gamma(T M),
$$

where $\beta=\xi(\varphi)-2 \varphi \tau(\xi)$. Thus, we have the following classification theorem for screen conformal lightlike hypersurface.

Theorem 3.3. Let $(M, g, S(T M))$ be an $(m+1)$-dimensional screen conformal lightlike hypersurface of a semi-Riemannian space form $(\bar{M}(c), \bar{g})$ and $m>2$. Then

(1) if $\beta=0$, we have $c=0$,

(2) if $\beta \neq 0$, we also have $c=0$. Moreover, in this case $S(T M)$ and $M$ are totally geodesic immersed in $M$ and $\bar{M}(c)$ respectively.

Proof. If $\beta=0$, from (3.7) it is easy to see $c=0$. Now we assume that $\beta \neq 0$. If $c \neq 0$, from (3.2) and (3.7) we know that

$$
B(X, Y)=\frac{c}{\beta} g(X, Y) \text { and } C(X, P Y)=\frac{\varphi c}{\beta} g(X, P Y),
$$

which means that both $S(T M)$ and $M$ are totally umbilical. By applying the Lemma 3.3 we get $B=0$ or $C=0$, which is equivalent to $c=0$ or $\varphi=0$ by using (3.8). Notice that $\varphi=0$ implies that $\beta=0$, there is a contradiction to the assumption. Thus we have $c=0$ and $B=C=0$ which completes the proof.

For a non-zero constant $\varphi$, we have $\xi(\varphi)=0$. So we have the following corollary following from Theorem 3.3.

Corollary 3.4. Let $(M, g, S(T M))$ be an $(m+1)$-dimensional screen homothetic lightlike hypersurface of a semi-Riemannian space form $(\bar{M}(c), \bar{g})$ and $m>2$. Then

(1) if $\tau(\xi)=0$, we have $c=0$, 
(2) if $\tau(\xi) \neq 0$, we have $c=0$. Moreover, in this case $S(T M)$ and $M$ are totally geodesic immersed in $M$ and $\bar{M}(c)$ respectively.

Recall the following notion of null sectional curvature following from $[2,3]$. Let $x \in M$ and $\xi$ be a null vector of $T_{x} M$. A plane $\mathrm{H}$ of $T_{x} M$ is called a null plane directed by $\xi$ if it contains $\xi, g_{x}(\xi, W)=0$ for any $W \in H$ and there exists $W_{o} \in H$ such that $g_{x}\left(W_{o}, W_{o}\right) \neq 0$. Thus the null section curvature of $H$ with respective to $\xi$ and the induced connection $\nabla$ of $M$, is defined as a real number

$$
K_{\xi}(H)=\frac{g_{x}(R(W, \xi) \xi, W)}{g_{x}(W, W)},
$$

where $W \neq 0$ is any vector in $H$ independent with $\xi$. Note that the authors in [10] proved that an $n(n \geq 3)$-dimensional Lorentzian manifold is of constant curvature if and only if its null sectional curvatures are everywhere zero.

Theorem 3.5. Let $(M, g, S(T M)$ be a screen conformal lightlike hypersurface of the semi-Riemannian space form $(\bar{M}(c), \bar{g})$, then the null sectional curvature of $M$ vanishes.

Proof. It follows from (2.13) that

$$
\begin{aligned}
& g(R(X, Y) Z, P W)=\bar{g}(\bar{R}(X, Y) Z, P W)+B(Y, Z) C(X, P W) \\
& \quad-B(X, Z) C(Y, P W)=\varphi(B(Y, Z) B(X, W)-B(X, Z) B(Y, W)) .
\end{aligned}
$$

From (2.8), we obtain $K_{\xi}(H)=0$. Then the proof is complete.

\section{Conformal Killing Distribution}

We need the following definition given by D. H. Jin in [7].

Definition 4.1. [7] Let $M$ be a semi-Riemannian manifold, a vector field $X$ on $M$ is said to be a conformal Killing vector field if $\mathcal{L}_{X} g=2 \alpha g$ for smooth function $\alpha$, where $\mathcal{L}$ denotes the Lie derivative with respect to $X$.

Let $(M, g)$ be a semi-Riemannian space form, then it follows from the above definition that

$$
X(\bar{g}(Y, Z))-\bar{g}([X, Y], Z)-\bar{g}(Y,[X, Z])=2 \alpha \bar{g}(Y, Z)
$$

for any $X, Y, Z \in \Gamma(T M)$. In particular, $X$ is called a Killing vector field if $\alpha=0$. A distribution $\mathcal{G}$ on $M$ is said to be a conformal Killing distribution if each vector field belonging to $\mathcal{G}$ is a conformal Killing vector field.

Theorem 4.2. Let $(M, g, S(T M))$ be a lightlike hypersurface of a semiRiemannian space form $(\bar{M}, \bar{g})$. If $\operatorname{Rad}(T M)$ is a conformal Killing distribution, i.e., $\mathcal{L}_{\xi} \bar{g}=2 \alpha \bar{g}$ for $\xi \in \Gamma(\operatorname{Rad}(T M))$, then we have

$$
B(X, Y)=-\alpha g(X, Y), \quad \forall X, Y \in \Gamma(T M),
$$


where $\alpha$ is a smooth function on $M$.

Proof. Notice that $\nabla$ is torsion-free connection, then it follows from (4.1) that

$$
\begin{aligned}
2 \alpha \bar{g}(Y, Z) & =\bar{g}\left(\bar{\nabla}_{X} \xi, Z\right)+\bar{g}\left(\bar{\nabla}_{Z} \xi, Y\right) \\
& =-g\left(A_{\xi}^{*} Y, Z\right)-g\left(A_{\xi}^{*} Z, Y\right), \quad \forall Y, Z \in \Gamma(T M) .
\end{aligned}
$$

Since $B$ is symmetric on $\Gamma(T M)$, then we have $B(X, Y)=-\alpha g(X, Y)$ following from the above equation. Thus, the proof is complete.

Theorem 4.3. Let $(M, g, S(T M))$ be a lightlike hypersurface of a semiRiemannian space form $(\bar{M}, g)$. If $\operatorname{Rad}(T M)$ is a conformal Killing distribution, then the smooth function $\alpha$ satisfies the following equation

$$
\xi(\alpha)+\alpha^{2}+\alpha \tau(\xi)=0 .
$$

Proof. Differentiating (4.2) gives

$$
\left(\nabla_{X} B\right)(Y, Z)=-X(\alpha) g(Y, Z)-\alpha\left(\nabla_{X} g\right)(Y, Z) .
$$

Substituting (2.9) and (2.14) into (4.5) and using (4.2), we have

$$
\begin{aligned}
& B(X, Z) \tau(Y)-B(Y, Z) \tau(X) \\
= & Y(\alpha) g(X, Z)-X(\alpha) g(Y, Z)+\alpha(B(Y, Z) \eta(X)-B(X, Z) \eta(Y)) .
\end{aligned}
$$

It follows from (4.6) and (4.2) that

$g(X, Z)\left(Y(\alpha)+\alpha^{2} \eta(Y)+\alpha \tau(Y)\right)-g(Y, Z)\left(X(\alpha)+\alpha^{2} \eta(X)+\alpha \tau(X)\right)=0$.

Replacing $Y$ by $\xi$ in the above equation, we have

$$
g(X, Z)\left(\xi(\alpha)+\alpha^{2}+\alpha \tau(\xi)\right)=0, \quad \forall X, Z \in \Gamma(T M) .
$$

Thus, the proof is complete.

Corollary 4.4. Let $(M, g, S(T M))$ be a lightlike hypersurface of a semiRiemannian space form $(\bar{M}, g)$ with a conformal Killing radical distribution. If $\alpha$ is a constant, then either $\alpha=0$ or $\alpha=-\tau(\xi)$.

Proof. Using Theorem 4.3 and the assumption that $\alpha$ is a constant, we have $\alpha^{2}+\alpha \tau(\xi)=\alpha(\alpha+\tau(\xi))=0$.

Lemma 4.5. Let $(M, g, S(T M))$ be a lightlike hypersurface of a semi-Riemannian space form $(\bar{M}, \bar{g})$. If $\operatorname{tr}(T M)$ is a conformal Killing distribution, i.e., $\mathcal{L}_{N} g=2 \rho g$ for $N \in \Gamma(\operatorname{tr}(T M))$, then we have

$$
C(X, Y)+C(Y, Z)=-2 \rho g(X, Y), \quad \forall X, Y \in \Gamma(S(T M)),
$$

where $\rho$ is a smooth function on $M$. 
Proof. Notice that $\nabla$ is torsion-free connection, then it follows from (4.1) that

$$
\begin{aligned}
2 \rho \bar{g}(Y, Z) & =\bar{g}\left(\bar{\nabla}_{Y} N, Z\right)+\bar{g}\left(\bar{\nabla}_{Z} N, Y\right) \\
& =-g\left(A_{N} Y, Z\right)-g\left(A_{N} Z, Y\right)+\tau(Y) \eta(X)+\tau(Z) \eta(Y),
\end{aligned}
$$

for any vector fields $X, Y, Z \in \Gamma(T M)$. By using (2.11) then we have $C(Y, P Z)+C(Z, P Y)=-2 \rho g(X, Y)+\tau(Y) \eta(Z)+\tau(Z) \eta(Y)$ following from the above equation. Thus, the proof is complete.

Corollary 4.6. Let $(M, g, S(T M))$ be a lightlike hypersurface of a semiRiemannian space form $(\bar{M}, \bar{g})$ with a conformal Killing transversal distribution, i.e., $\mathcal{L}_{N} g=2 \rho g$ for $N \in \Gamma(\operatorname{tr}(T M))$. If $S(T M)$ is integrable, then we have

$$
C(X, Y)=-\rho g(X, Y), \quad \forall X, Y \in \Gamma(S(T M)) .
$$

Proof. Notice that $\nabla$ is torsion-free connection, then it follows from (2.5) that

$$
\begin{aligned}
\bar{g}(N,[X, Y]) & =\bar{g}\left(\bar{\nabla}_{X} N, Y\right)-\bar{g}\left(\bar{\nabla}_{Y} N, X\right) \\
& =g\left(A_{N} Y, X\right)-g\left(A_{N} X, Y\right), \quad \forall X, Y \in \Gamma(S(T M)) .
\end{aligned}
$$

If $S(T M)$ is integrable, from (4.12) we know that $C$ is symmetric on $S(T M)$. Thus, we complete the proof by using (4.9).

Note that $C(\xi, P X)=g\left(A_{N} \xi, P X\right)$, then we have the following corollary.

Corollary 4.7. Let $(M, g, S(T M))$ be a lightlike hypersurface of a semiRiemannian space form $(\bar{M}, \bar{g})$ with a conformal Killing transversal distribution. If $S(T M)$ is integrable and $A_{N} \xi=0$, then $S(T M)$ is totally umbilical.

Theorem 4.8. Let $(M, g, S(T M))$ be a lightlike hypersurface of a semiRiemannian space form $(\bar{M}, \bar{g})$ with a conformal Killing transversal distribution, i.e., $\mathcal{L}_{N} g=2 \rho g$ for $N \in \Gamma(\operatorname{tr}(T M))$. If $S(T M)$ is integrable, then we have

$$
\xi(\rho)-\rho \tau(\xi)=0
$$

where $\rho$ is a smooth function on $M$.

Proof. By differentiating (4.11), then for any $X, Y, Z \in \Gamma(S(T M))$ we have

$$
\left(\nabla_{X} C\right)(Y, Z)=-X(\rho) g(Y, Z)-\rho\left(\nabla_{X} g\right)(Y, Z) .
$$

Substituting (2.9) and (2.16) into (4.14) and using (4.2), we have

$$
g(X, Z)(Y(\rho)-\rho \tau(Y))-g(Y, Z)(X(\rho)-\rho \tau(X))=0 .
$$

Replacing $\mathrm{Y}$ by $\xi$ in the above equation, we obtain

$$
g(X, Z)(\xi(\rho)-\rho \tau(\xi))=0, \quad \forall X, Z \in \Gamma(S(T M)) .
$$


Thus, the proof is complete.

Acknowledgements. Both authors wish to thank the referee for the valuable suggestions in the improvement of the paper.

\title{
REFERENCES
}

[1] C. Atindogbe and K. L. Duggal, Conformal screen on lightlike hypersurfaces, Int. J. Pure Appl. Math., 11 (4) (2004), 421-442.

[2] A. Bejancu and K. L. Duggal, Degenerate hypersurface of semi-Riemannian manifolds, Bull. Inst. Politehnie Iasi, 37 (1991), 13-22.

[3] K. L. Duggal and A. Bejancu, Lightlike Submainfolds of Semi-Riemannian Mainfolds and Applications, Kluer Academic Publishers, Dordrecht, 1996.

[4] K. L. Duggal and D. H. Jin, Null Curves and Hypersurfaces of Semi-Riemannian Manifolds, World Scientific, 2007.

[5] K. L. Duggal and B. Sahin, Differential Geometry of Lightlike Submanifolds, Birkhauser Veralag AG, Basel, Boston, Berlin, 2010.

[6] D. H. Jin, Lightlike hypersurface with totally umbilical screen distribution, J. Chungcheong Math. Soc., 22 (3) (2009), 409-415.

[7] D. H. Jin, Geometry of half lightlike submanifolds of a semi-Riemannian space form with a semi-symmetric metric connection, J. Chungcheong Math. Soc., 24 (4) (2011), 769-780.

[8] D. H. Jin and J. W. Lee, Einstein half lightlike submanifolds of a Lorentzian space form with a semi-symmetric metric connection, to appear in Mediterranean J. Math.

[9] D. N. Kupeli, Singular semi-Riemnnian Geometry, Mathematics and Its Applications, Kluwer Academic Publishers, Dordrecht, 1996.

[10] B. O'Neill, Semi-Riemannian Geometry with Application to Relativity, Academic Press, New York, 1983.

(Received: September 16, 2012)

\author{
Yaning Wang \\ Department of Mathematics \\ South China University of Technology \\ Guangzhou 510641, Guangdong \\ P. R. China \\ wyn051@163.com \\ Ximin Liu \\ School of Mathematical Sciences \\ Dalian University of Technology \\ Dalian 116024, Liaoning \\ P. R. China \\ ximinliu@dlut.edu.cn
}

